PROCEEDINGS OF THE

AMERICAN MATHEMATICAL SOCIETY

Volume 126, Number 9, September 1998, Pages 2755-2762

S $0002-9939(98) 04510-9$

\title{
HALF-SPACE THEOREMS FOR MEAN CURVATURE ONE SURFACES IN HYPERBOLIC SPACE
}

\author{
LUCIO RODRIGUEZ AND HAROLD ROSENBERG
}

(Communicated by Peter Li)

\begin{abstract}
We give conditions which oblige properly embedded constant mean curvature one surfaces in hyperbolic 3-space to intersect. Our results are inspired by the theorem that two disjoint properly immersed minimal surfaces in $\mathbf{R}^{3}$ must be planes.
\end{abstract}

The half-space theorem says that a properly immersed minimal surface in $\mathbf{R}^{3}$ that is disjoint from a plane (thus in a half-space) is a plane. The strong halfspace theorem says that two disjoint properly immersed minimal surfaces in $\mathbf{R}^{3}$ are planes. The latter is deduced from the former by finding a plane between the two surfaces. These theorems are due to D. Hoffman and W. Meeks [H-M].

In this paper we establish results of this nature in hyperbolic 3-space for mean curvature one surfaces and horospheres.

Let $N$ denote a horosphere of $\mathbf{H}^{3}$, and $B$ the horoball of $\mathbf{H}^{3}$ bounded by $N$; the mean curvature of $N$ is one and the mean curvature vector of $N$ points into $B$. Let $C$ be the other connected component of $\mathbf{H}^{3} \backslash N$.

Theorem 1. Let $M$ be a properly embedded constant mean curvature one surface in $\mathbf{H}^{3}$, and assume $M \cap N=\varnothing$. If $M \subset B$, then $M$ is a horosphere. If $M \subset C$ and the mean curvature vector of $M$ points towards $N$ (i.e. it points into the component of $\mathbf{H}^{3}$ bounded by $\left.N \cup M\right)$, then $M$ is a horosphere.

In the same spirit we shall establish:

Theorem 2. Let $N$ be a catenoid cousin [B] (this is presented in section 1) and let $B$ be the connected component of $\mathbf{H}^{3}$ to which the mean curvature vector of $N$ points (that is, $B$ is the mean convex domain bounded by $N$ ). Let $M$ be a properly embedded constant mean curvature one surface in $\mathbf{H}^{3}$, disjoint from $N$. Then $M \cap B=\varnothing$ and the mean curvature vector of $M$ does not point towards $N$ (notice that such $M$ exist, e.g., any horosphere in $\mathbf{H}^{3} \backslash \bar{B}$ ).

Theorem 3. Let $M_{1}$ and $M_{2}$ be disjoint properly embedded constant mean curvature one surfaces in $\mathbf{H}^{3}$. Let $W$ be the connected domain of $\mathbf{H}^{3}$ bounded by $M_{1}$ and $M_{2}$. Then $W$ is not mean convex, i.e. the mean curvature vectors of $M_{1}$ and $M_{2}$ do not both point into $W$.

Received by the editors September 10, 1996.

1991 Mathematics Subject Classification. Primary 53A10.

(C)1998 American Mathematical Society 


\section{Preliminaries}

The proofs of the theorems are inspired by the proof of Hoffman-Meeks. Minimal surfaces in $\mathbf{R}^{3}$ are locally isometric to constant mean curvature one surfaces in $\mathbf{H}^{3}$ and in many ways they behave analogously [B]. The half-space theorem in $\mathbf{R}^{3}$ uses a catenoid and its' homotheties. In $\mathbf{H}^{3}$, a one-parameter family of catenoid cousins plays the same role.

The catenoid cousins are rotational embedded constant mean curvature one surfaces. Take for $\mathbf{H}^{3}$ the model $\left\{x_{3} \geq 0\right\} \subset \mathbf{R}^{3}$ and let $I$ be the geodesic of $\mathbf{H}^{3}$ corresponding to the positive $x_{3}$-axis. The catenoid cousins with rotational axis $I$ and waist at height $c>0$ are a one-parameter family $N(t), 0<t<\infty$, of constant mean curvature one surfaces, each homeomorphic to $S^{1} \times \mathbf{R}$ having the following properties:

i) As $t \rightarrow 0, N(t)$ converges to two horospheres meeting at the point $(0,0, c)$, one horosphere is the horizontal plane at height $c$ and the other is the sphere centered at $(0,0, c / 2)$, of (euclidean) radius $c / 2$; see Figure $1(\mathrm{a}),(\mathrm{b})$.

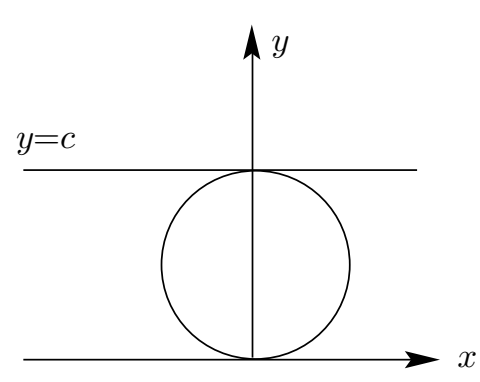

(a)

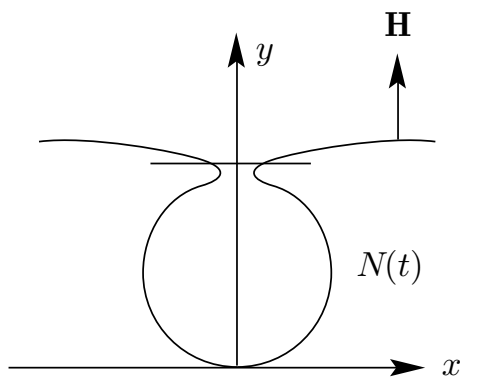

(b)

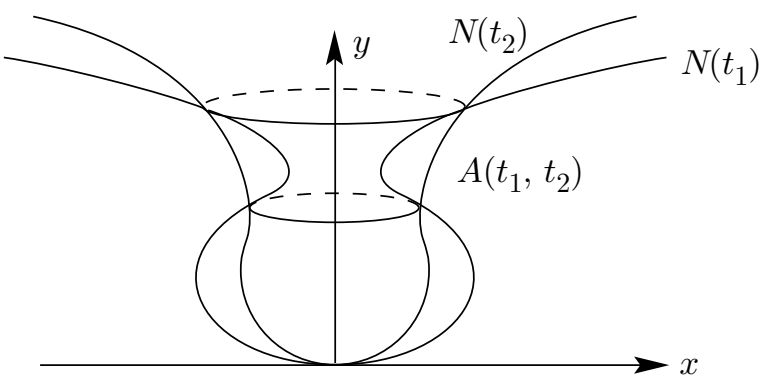

(c)

FigURE 1

ii) For $t_{1} \neq t_{2}, t_{1}, t_{2}>0, N\left(t_{1}\right) \cap N\left(t_{2}\right)$ consists of two (horizontal) circles $c\left(t_{1}\right)$ and $c\left(t_{2}\right)$.

iii) For $t_{1}<t_{2}, c\left(t_{1}\right) \cup c\left(t_{2}\right)$ bounds a compact annulus $A\left(t_{1}, t_{2}\right)$ contained in $N\left(t_{2}\right)$. As $t$ varies from $t_{1}$ to infinity, the annuli $A\left(t_{1}, t\right)$ exhaust $C\left(t_{1}\right)$, where $C\left(t_{1}\right)$ is the connected component of $\mathbf{H}^{3} \backslash N\left(t_{1}\right)$ which does not contain $I$; see Figure 1(c).

iv) Fix $t>0$ and let $h(r)=x_{3}$ be the height (euclidean will do) of $N(t)$, for $r^{2}=x_{1}^{2}+x_{2}^{2}$ (this makes sense for $r$ large). Then $h(r) \rightarrow \infty$ as $r \rightarrow \infty$. 
For each $c>0$, there is a family $N(t)$ of catenoid cousins at waist height $c$. In fact, euclidean homothety from $(0,0,0)$ is a hyperbolic isometry and these isometries act transitively on $I$. The properties i) through iv) will be established in an appendix.

Any constant mean curvature one rotational surface that is embedded and has axis $I$ is one of the catenoid cousins $N(t)$ for a certain waist height $c$.

\section{Proofs of the theorems}

Proof of Theorem 1. Let $M$ be a properly embedded constant mean curvature hypersurface in hyperbolic $n$-space whose asymptotic boundary at infinity is at most two points. Then it is known that $M$ is a rotational hypersurface about the geodesic joining the two points [L-R]. When the asymptotic boundary of such an $M$ is one point, do Carmo and Lawson proved $M$ is a horosphere [doC-L].

Now let $N$ be a horosphere and $M$ a constant mean curvature one surface in $\mathbf{H}^{3}$, properly embedded. We assume $M \cap N=\varnothing$. If $M$ is contained in the horoball $B$ bounded by $N$, then the asymptotic boundary of $M$ is one point, the point at infinity of $N$. Thus $M$ is a horosphere.

So assume $M \subset C=\mathbf{H}^{3} \backslash \bar{B}$ and the mean curvature vector of $M$ points towards $N$. We work in the upper half-space model of $\mathbf{H}^{3}$. Let $N$ be the horosphere which is the horizontal plane at height $c_{0}$. Then $M \subset\left\{0<x_{3}<c_{0}\right\}$. By the usual maximum principle (and our hypothesis on the direction of the mean curvature vector of $M$ ) we can assume $M$ is asymptotic to $N$ at infinity, i.e. there exists a sequence $p_{n} \in M$ such that $r\left(p_{n}\right) \rightarrow \infty$ as $n \rightarrow \infty$, and $x_{3}\left(p_{n}\right) \rightarrow c_{0}$ (more precisely, lower $N$ until it touches $M$ for the first time; if this touching point is not at infinity, then $M$ equals the lowered horizontal plane); we recall that $r^{2}=x_{1}^{2}+x_{2}^{3}$. Since $M$ is properly embedded, no point of $N$ is an accumulation point of $N$. Let $\varepsilon>0$ be chosen so that the ball of radius $\varepsilon$ centered at $\left(0,0, c_{0}\right)$ does not meet $M$. Let $c=c_{0}-\varepsilon / 2$ and consider the family of catenoid cousins $N(t)$ with the waist height $c$. Denote by $A(t)$ the part of $N(t)$ above height $c$.

By property i) of the family $N(t), A(t)$ converges to the horosphere $\left\{x_{3}=c\right\}$ as $t \rightarrow 0$. Also $\partial A(t) \rightarrow(0,0, c)$ as $t \rightarrow 0$. By property iv), the height of $A(t)$ is unbounded.

Now let $\varepsilon$ and $t_{0}$ be close enough to zero so that $A\left(t_{0}\right) \cap M=\varnothing$. As $t$ varies from $t_{0}$ to $0, A(t)$ converges to $\left\{x_{3}=c\right\}$, uniformly on compact sets. There are points of $M$ above height $c$ (strictly) and $M$ is properly embedded, so there is a first value of $t$ (largest $t$ in $\left.\left(0, t_{0}\right)\right)$ such that $A(t) \cap M \neq \varnothing(A(t)$ does not first touch $M$ at infinity since the height of $A(t)$ is unbounded). The mean curvatures of $A(t)$ and $M$ point in the same direction at the point where they touch, so $M=A(t)$ by the maximum principle. This is impossible since $A(t)$ meets $N$ and $M$ is disjoint from $N$; see Figure 2.

Proof of Theorem 2. First we show that $M \cap B=\varnothing$. If not, then $M \subset B$ so the asymptotic boundary of $M$ is at most two points, the points at infinity of $N$. By the theorem of Levitt and Rosenberg which we cited in the first paragraph of the proof of Theorem 1, $M$ is a rotational surface with $I$ as axis [L-R]. Thus $M$ is a horosphere or a catenoid cousin. But there is no horosphere or catenoid cousin inside $N$. For the horosphere one can see this directly or apply Theorem 1 (which would oblige $N$ to be a horosphere). Any two catenoid cousins with the same 


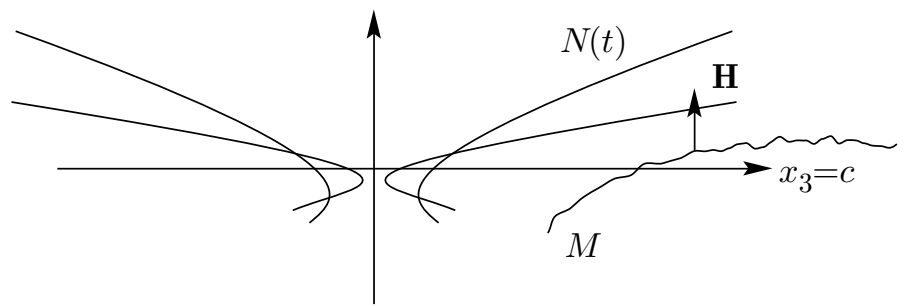

Figure 2

axis intersect; this is property ii) when they have the same waist height, and in general they differ by a homothety from $(0,0,0)$. So it remains to show that when $M \subset C=\mathbf{H}^{3} \backslash B$, then the mean curvature vector of $M$ does not point towards $N$.

We use property iv). Let $N=N\left(t_{1}\right)$ and consider the compact annuli $A\left(t_{1}, t\right)$ as $t$ goes from $t_{1}$ to infinity. $M$ is properly embedded, so for $t$ small, $A\left(t_{1}, t\right)$ is disjoint from $M$. As $t$ increases there is a first value of $t$ where $A\left(t_{1}, t\right)$ touches $M$. At this point, the mean curvature vectors of $M$ and $A\left(t_{1}, t\right)$ coincide, $\partial A\left(t_{1}, t\right) \subset N$ so the touching point is an interior point of $A\left(t_{1}, t\right)$ and the maximum principle then implies $M$ is a catenoid cousin. This is impossible since $M \cap N=\varnothing$.

Proof of Theorem 3. Let $\gamma$ be an arc from a point $q_{1} \in M_{1}$ to a point $q_{2} \in M_{2}$. Let $E_{n} \subset E_{n+1}$ be an exhaustion of $M_{1}$ by compact connected submanifolds with boundary $E_{n}$ equal to $\Gamma_{n}$. Also suppose $q_{1} \in E_{1}$. Let $\Sigma_{n}$ be a solution to the $H=1$ Plateau problem with $\partial \Sigma_{n}=\Gamma_{n}, \Sigma_{n} \subset W$, and $\Sigma_{n}$ homologous to $E_{n}$ relative to $\Gamma_{n}$ in $W$. One obtains $\Sigma_{n}$ by minimizing the functional $\left|\tilde{\Sigma}_{n}\right|+2|Q|$ where $Q$ is a domain with $\partial Q=E_{n}+\tilde{\Sigma}_{n}$. $\partial W$ is a good barrier, so a minimum exists and is smooth $[\mathrm{A}-\mathrm{R}]$.

Each $\Sigma_{n}$ intersects $\gamma$ since the linking number is a homology invariant, so a subsequence of the $\Sigma_{n}$ converges to a stable $H=1$ surface $\Sigma$ in $W$.

By a theorem of Silveira, the horosphere is the only complete stable $H=1$ surface in $\mathbf{H}^{3}$ [S], so $\Sigma$ is a horosphere and then so are $M_{1}$ and $M_{2}$ by Theorem 1. This is impossible since two disjoint horospheres do not bound a mean convex domain.

Remarks. 1. It seems that if $M_{1}$ and $M_{2}$ are properly embedded mean curvature one surfaces in $\mathbf{H}^{3}$ and if the mean curvature vector of $M_{1}$ points towards $M_{2}$, $M_{1} \cap M_{2}=\varnothing$, then $M_{1}$ and $M_{2}$ are horospheres.

2. Is there any properly embedded mean curvature one surface in $\mathbf{H}^{3}$ that is simply connected and different from the horosphere? In $\mathbf{R}^{3}$, the helicoid and plane exist.

3. Is there a properly embedded mean curvature one annulus in $\mathbf{H}^{3}$, other than a catenoid cousin? First, one could try to decide this for such $M$ that are transverse to the horospheres $x_{3}=$ constant. The part of the annulus above a horosphere is conformally a punctured disk since $\ln x_{3}$ is superharmonic on the surface. In $\mathbf{R}^{3}$, the only properly embedded minimal annulus is a catenoid. 


\section{APPENDix}

In this section we will prove the properties of constant mean curvature one surfaces of revolution. First, it is known that the distance from the generating curve to the rotation axis attains a minimum at a unique point.

For the half-space model of $\mathbf{H}^{3}$, where the orbit space is the two-dimensional halfplane one rotates the generating curve about the geodesic $x=0$, and the generating curve has coordinates $(x(s), y(s))$ parametrized by arc-length with respect to the metric of $\mathbf{R}^{2}$. Any curve in $\mathbf{H}^{2}$ parametrized by arc-length with respect to the metric of $\mathbf{R}^{2}$ has geodesic curvature $k_{g}$ in $\mathbf{H}^{2}$ given by $k_{g}=y(s) \alpha^{\prime}(s)+\cos (\alpha(s))$, where $\alpha(s)$ denotes the angle the curve makes with the $x$-axis. Thus, the principal curvatures are given by

$$
k_{1}=y(s) \alpha^{\prime}(s)+\cos (\alpha(s))
$$

and

$$
k_{2}=\cos (\alpha(s))+\frac{y(s)}{x(s)} \sin (\alpha(s)) .
$$

Hence the generating curves for a rotational surface of constant curvature $H$ satisfy the following system of differential equations:

$$
\begin{aligned}
x^{\prime}(s) & =\cos (\alpha(s)), \\
y^{\prime}(s) & =\sin (\alpha(s)), \\
\alpha^{\prime}(s) & =\frac{2 H-2 \cos (\alpha(s))-\frac{y(s)}{x(s)} \sin (\alpha(s))}{y(s)} .
\end{aligned}
$$

Now using the last equation we have

$$
y(s)\left(\alpha^{\prime}(s)+\frac{\sin (\alpha(s))}{x(s)}\right)=2 H-2 \cos (\alpha(s)) .
$$

To prove property iv), we want to show first that $y(s)$ is unbounded as $s \rightarrow \infty$. We observe that the generating curve is tangent to the line $y=\lambda_{0} x, \lambda_{0}>0$, at the waist point $\left(x\left(s_{0}\right), y\left(s_{0}\right)\right)$. At this point $y^{\prime}\left(s_{0}\right)$ and $x^{\prime}\left(s_{0}\right)$ are positive and $\alpha^{\prime}\left(s_{0}\right)<0$. We have that $y^{\prime}(s) \geq 0$ for all $s \geq s_{0}$; otherwise a piece of the generating curve will be below the level $y \equiv b$ for some constant $b$; using the maximum principle with the horosphere $y \equiv b$ gives a contradiction. Also if $x^{\prime}(s)$ or $\alpha^{\prime}(s)$ changes sign for some $s>s_{0}$, then it is clear that a piece of the generating curve is above the line $y=a+\lambda_{1} x, \lambda_{1}>0, a>0$. But the cone of revolution of this line $(x>0)$ has $H>1$, and the maximum principle gives a contradiction. Since the right hand side of the above equation is positive when $H \geq 1$, we must have that

$$
\alpha^{\prime}(s)+\frac{\sin (\alpha(s))}{x(s)} \geq 0 .
$$

We get, using the first equation of the system, that

$$
\frac{x^{\prime}(s)}{x(s)} \geq \frac{-\alpha^{\prime}(s) \cos \alpha(s)}{\sin \alpha(s)} .
$$

By making the change of variable $u(s)=\sin \alpha(s)$, we obtain

$$
\log x(s)-\log x\left(s_{0}\right) \geq-\log (u(s))-\log \left(u\left(s_{0}\right)\right) .
$$


Taking the exponential of this expression we obtain

$$
x(s) \geq \frac{C}{u(s)}=\frac{C}{\sin (\alpha(s))},
$$

where $C=x\left(s_{0}\right) u\left(s_{0}\right)$. Thus, $-1 / x(s) \geq \frac{\sin (\alpha(s))}{-C}$. If we further assume by contradiction that $y(s) \leq \widetilde{C}$, then we obtain that $-\frac{y(s)}{x(s)} \geq-D \sin (\alpha(s))$, where $D=\frac{\widetilde{C}}{C}>0$. Now the third equation of the system gives us that

$$
\begin{aligned}
y(s) \alpha^{\prime}(s) & \geq 2-2 \cos (\alpha(s))-D \sin ^{2}(\alpha(s)) \\
& =2-2 \cos (\alpha(s))-D\left(1-\cos ^{2} \alpha(s)\right) \\
& \geq 2-2 \cos (\alpha(s))-2 D(1-\cos \alpha(s))
\end{aligned}
$$

since $-D\left(1-\cos ^{2} \alpha(s)\right)=-D(1-\cos \alpha(s))(1+\cos \alpha(s)) \geq-2 D(1-\cos \alpha(s))$. Since $\alpha^{\prime}<0$, so is the right hand side of the above equation, and we obtain, using the second equation of the system, that

$$
\frac{\alpha^{\prime}(s) \sin \alpha(s)}{2-2 \cos (\alpha(s))-2 D(1-\cos \alpha(s))} \leq \frac{y^{\prime}(s)}{y(s)} .
$$

Now, by the change of variable $u(s)=\cos \alpha(s)$, we obtain that

$$
\begin{aligned}
\log \left(y\left(s_{1}\right)\right)-\log \left(y\left(s_{0}\right)\right) & =\int_{s_{0}}^{s_{1}} \frac{y^{\prime}(s) d s}{y(s)} \\
& \geq \int_{\cos \alpha\left(s_{0}\right)}^{\cos \alpha\left(s_{1}\right)} \frac{-d u}{(2-2 D)(1-u)} \\
& =-\left.\frac{\log (1-u)}{2 D-2}\right|_{\cos \alpha\left(s_{0}\right)} ^{\cos \alpha\left(s_{1}\right)} .
\end{aligned}
$$

If $D>1$, this integral goes to $\infty$ as $s \rightarrow \infty$. Certainly, we can make $D$ bigger by taking a bigger upper bound $\widetilde{C}$ for $y(s)$. This proves property iv).

It is more convenient to now work in the ball model of $\mathbf{H}^{3}$ where the generating curve is again denoted $(x(s), y(s))$ and the surface is obtained by rotating around the $x$-axis. $x$ measures hyperbolic distance along the rotation axis and $y$ is the hyperbolic distance of the point on the profile curve to the $x$-axis. In this coordinate system the hyperbolic metric is given by

$$
\cosh ^{2}(y) d s^{2}+d y^{2}
$$

Castillon $[\mathrm{C}]$ using this coordinate system proved a beautiful theorem describing these surfaces of revolution as coming from a rolling construction. He found a first integral of the differential equation that these surfaces must satisfy:

$$
\frac{d x}{d s}=\frac{\sin \alpha}{\cosh y}, \quad \frac{d y}{d s}=\cos \alpha .
$$

The principal curvatures are given by

$$
k_{1}=\frac{d \alpha}{d s}+\sin \alpha \tanh y, \quad k_{2}=\sin \alpha \operatorname{coth} y .
$$

The mean curvature is given by

$$
2 H=\frac{d \alpha}{d s}+\sin \alpha(\tanh y+\operatorname{coth} y) .
$$


Then the first integral in terms of $y$ and $\alpha$ is:

$$
F(y, \alpha)=-H \sinh ^{2} y+\sinh y \cosh y \sin \alpha .
$$

Thus, on a profile curve the $F(y, \alpha)$ is equal to a constant $C$. Now this constant is related to the point closest to the $x$-axis, where $\alpha=\pi / 2$ and

$$
C=-\sinh ^{2} y+\sinh y \cosh y .
$$

Observe that as this minimum distance varies from zero to infinity, $C$ varies from 0 to $\frac{1}{2}$. Also, the derivative of $C$ in terms of $y$ at this minimum point is

$$
\cosh 2 y-\sinh 2 y>0 \text {. }
$$

Thus $C$ is monotonically increasing in terms of the minimum distance to the origin.

We also need the following facts. The point $y_{C}(0)$ is a local minimum of the profile curve. Also because of the comparison principle (by this we mean that if $M_{2}$ is locally on one side of $M_{1}$ at a point $x \in M_{1} \cap M_{2}$ and if the mean curvature vector of $M_{1}$ points to the side where $M_{2}$ is at $x$, then $H_{M_{2}} \geq H_{M_{1}}$ ), at no other point does the distance to the rotation axis have a local maximum; thus $y$ is an increasing function of $x$, for $x \geq 0$.

Observe that as $C$ increases to $\frac{1}{2}$ the minimum distance of the profile curve to the $x$-axis goes to infinity (since $y$ is monotone increasing for $x \geq 0$ ).

Let $y_{C_{1}}(0)$ and $y_{C_{2}}(0)$ be the points on the profile curves corresponding to the solutions $y_{C_{1}}$ and $y_{C_{2}}$ at $x=0$. Then letting $C_{2}$ increase to $\frac{1}{2}$ and keeping $C_{1}$ fixed, the intersection points of the profile curves $y_{C_{1}}(x)$ and $y_{C_{2}}(x)$ (if they exist) are at least a distance $y_{C_{2}}(0)$ from the $x$-axis. So to establish property iii), it suffices to show the two profile curves intersect.

By (1), we have that $\frac{d x}{d y}=\frac{\tan \alpha(y)}{\cosh y}$. By (2), we have that $\sin \alpha(y)=\frac{C+\sinh ^{2} y}{\sinh y \cosh y}$. Since $\tan \left(\sin ^{-1}(w)\right)=\frac{w}{\sqrt{1-w^{2}}}$ we obtain that

$$
\begin{aligned}
\frac{d x}{d y} & =\frac{\tan \left(\sin ^{-1}\left(\frac{C+\sinh ^{2} y}{\sinh y \cosh y}\right)\right)}{\cosh y} \\
& =\frac{C+\sinh ^{2} y}{\cosh y \sqrt{\sinh ^{2} y \cosh ^{2} y-C^{2}-2 C \sinh ^{2} y-\sinh ^{4} y}} \\
& =\frac{C+\sinh ^{2} y}{\cosh y \sqrt{\sinh ^{2} y(1-2 C)-C^{2}}} \\
& =\frac{(C-1)+\cosh ^{2} y}{\cosh y \sqrt{\cosh ^{2} y(1-2 C)-(C-1)^{2}}} .
\end{aligned}
$$

Now observe that $\lim _{y \rightarrow \infty} \frac{d x}{d y}=\frac{1}{\sqrt{1-2 C}}>0$ if $C<\frac{1}{2}$.

Thus if $C_{1}<C_{2}$, we have $y_{C_{1}}(0)<y_{C_{2}}(0)$ while $y_{C_{1}}(x)>y_{C_{2}}(x)$ for $x$ large, so the curves intersect.

\section{REFERENCES}

[A-R] H. Alencar and H. Rosenberg, Some remarks on the existence of hypersurfaces of constant mean curvature with a given boundary, or asymptotic boundary, in hyperbolic space, Bull. des Sciences Maths. de France 121 (1997), 61-69.

[B] B. Bryant, Surfaces of mean curvature one in hyperbolic space, Astérisque 154-155 (1987), 341-347. 
[C] P. Castillon, Sur le surfaces de révolution à courbure moyenne constante dans l'espace hyperbolique, Preprint.

[doC-L] M. do Carmo and B. Lawson, On Alexander-Bernstein theorems in hyperbolic space, Duke Math. J. 50 (1983), 995-1003. MR 85f:53009

[H-M] D. Hoffman and W. Meeks, The strong half-space theorem for minimal surfaces, Invent. Math. 101 (1990), 373-377. MR 92e:53010

[L-R] G. Levitt and H. Rosenberg, Symmetries of constant mean curvature hypersurfaces in hyperbolic space, Duke Math. J. 52 (1985), 53-59. MR 86h:53063

[S] A. Silveira, Stability of complete noncompact surfaces with constant mean curvature, Math. Ann. 277 (1987), 629-638.

Institute for Pure-Applied Mathematics, Estrada Dona Castorina 110, 22460 Rio de JANEIRO, BRAZIL

E-mail address: lucio@impa.br

Department of Mathematics, University of Paris Vit, 2 place Jussieu, 75251 Paris, FRANCE

E-mail address: rosen@math.jussieu.fr 